\title{
El uso actual o reciente de anticonceptivos orales modifica el riesgo de muerte.
}

Mortality associated with oral contraceptive use: 25 year follow up of cohort of 46000 women from Royal College of General Practitioners' oral contraception study. Beral V, Hermon C, Kay C et al. BMJ. 1999;318: 96-100

\section{Objetivo}

Evaluar la influencia del uso de anticonceptivos orales (ACO) sobre la mortalidad.

\section{Diseño}

Estudio de cohortes de 25 años de seguimiento. Los datos acerca del uso de anticonceptivos orales y morbi-mortalidad fueron reportados semestralmente por médicos generales. El $75 \%$ de la cohorte original fue "marcada" en los registros centrales del Servicio Nacional de Salud (NHS) de Inglaterra y Escocia para obtener información sobre muerte y causa de muerte de aquellas mujeres que no pudieron ser seguidas con regularidad.

\section{Lugar}

Gran Bretaña.

\section{Participantes}

De las 46000 mujeres estudiadas, la mitad estaba usando ACO durante el reclutamiento en 1968/9. La mediana de la edad al final del seguimiento fue de 49 años.

\section{Medición de resultados principales}

Riesgo relativo (RR) de muerte ajustado para edad, paridad, clase social y hábito de fumar.

\section{Resultados principales}

Durante los 25 años de seguimiento fueron reportadas 1599 muertes. El riesgo de muerte para todas las causas fue similar en las que utilizaron y las que nunca utilizaron ACO (RR=1.0 [IC 95\% 0.9 a 1.1; $\mathrm{P}=0.7]$ ) y el riesgo de muerte por causas específicas no difirió significativamente en ambos grupos.

Sin embargo, entre las utilizadoras actuales o recientes (menos de 10 años de suspendida la toma) el RR de muerte para cáncer de ovario fue de 0.2 [0.1 a 0.8; $P=0.01]$, pará cáncer de cuello uterino 2.5 [1.1 a $6.1 ; P=0.04$ ], y para enfermedad cerebrovascular de 1.9 [1.2 a 3.1; $P=0.009]$. En contraste, las mujeres que habían suspendido el uso de ACO hacía mas de 10 años no tuvieron modificaciones en el RR de muerte para causas específicas ni para todas las causas.

\section{Conclusiones}

Los principales efectos sobre mortalidad se manifiestó en utilizadoras actuales o recientes (menos de 10 años). Aunque la mortalidad global fue similar, el uso de ACO se asoció a una disminución relativa de la muerte por cáncer de ovario y a un aumento relativo de las muertes por cáncer de cuello uterino, y enfermedad cerebrovascular. Luego de los 10 años de suspensión de la toma, la mortalidad fue similar en utilizadoras pasadas y en las que nunca utilizaron.

\section{COMENTARIO}

Desde su aparición en 1960, los AC0 han influído en la vida de millones de personas y han tenido un efecto revolucionario sobre la sociedad mundial.

Al ser empleados en población joven y generalmente saludable, la consideración de sus posibles efectos adversos tiene especial importancia.

Este trabajo intenta evaluar el riesgo de muerte asociado al uso de ACO en población británica, blanca, casada o que vivía en pareja y se consideraron los ACO que contenían 50 ug. de estrógeno; valor muy superior a los empleados en las formulaciones de las píldoras actuales (20 a $30 \mathrm{ug})$.

Aunque el incremento de mortalidad por enfermedad vascular (principalmente ACV) para usuarias actuales o recientes de ACO y, en particular si son fumadoras, sea controvertido con otros estudios clínicos de importancia en los que se utilizaron formulaciones con menor contenido estrogénico 1-2, existe coincidencia en sugerir el uso de ACO sin estrógenos para fumadoras mayores de 35 años?

En relación al aumento del riesgo para cáncer cervical, no esta aclarado si se controlaron en el estudio factores potencialmente confundidores *, como el número de parejas sexuales, la regularidad del ras- treo citológico (PAP) o el uso de métodos de barrera. Hasta el momento no existen estudios que puedan atribuir a los componentes hormonales de las píldoras un efecto causal en la neoplasia cervical. Sí es conocido el rol del HPV y del Herpes Simplex tipo II como inductores de la neoplasia cervical, por lo que el tipo de contracepción modifica las posibilidades de transmisión de estos agentes. ${ }^{3}$

En cuanto a la protección al riesgo de muerte por cáncer de ovario, los resultados de este estudio coinciden con los de otras investigaciones y con los conocimientos actuales al respecto, incrementando la fuerza de evidencia sobre este tema.

A pesar de estos cambios relativos en la mortalidad, hay que tener en cuenta que, dado la baja mortalidad basal de este grupo etario, el impacto absoluto de los ACO en la mortalidad es bajo.

La continuidad del seguimiento de ésta y otras cohortes proporcionará información clave para las recomendaciones y el seguimiento de miLlones de mujeres usuarias o potenciales usuarias de ACO. Excepto las fumadoras mayores de 35 años, en los que se aconsejan ACO sin estrógenos u otros métodos, las usuarias de las formulaciones de ACO con bajas dosis de estrógenos pueden quedarse tranquilas y seguir utilizando este método de alta efectividad y mucha seguridad.

*Ver Glosario

\section{Dr. Fabián Portnoy}

Servicio de Area Programática Hospital J.M. Penna.GCBA. S.P.M. (Sistema de Protección Médica)

\footnotetext{
Referencias

1. Colditz G. Oral Contraceptive Use and Mortality during 12 Years of Follow-Up: The Nurses Health Study. Ann Intern Med, vol 120 (10). May 15, 1994. pp821-826.

2. Schwingl P, Ory H, Visness C. Estimates of risk of cardiovascular death attributable to low-dose oral contraceptives in the United States. Am J 0bstet Gynecol, vol 180 (1). January 1999. pp 241-249.

3. Stergachis A. Epidemiology of the noncontraceptive effects of oral contraceptives. Am J Obstet Gynecol, 167. 1992. pp 1165-70.
} 